\title{
Investigating Immune System Aging: System Dynamics and Agent-Based Modeling
}

\author{
Grazziela P. Figueredo, Uwe Aickelin \\ IMA Research Group, Computer Science School, Nottingham University, Wollaton Road, Nottingham, NG8 1BB UK \\ gzf@cs.nott.ac.uk, uxa@cs.nott.ac.uk
}

Keywords: Immunosenescence, thymic output, naive T cell dynamics, system dynamics, agent based simulation

\begin{abstract}
System dynamics and agent based simulation models can both be used to model and understand interactions of entities within a population. Our modeling work presented here is concerned with understanding the suitability of the different types of simulation for the immune system aging problems and comparing their results. We are trying to answer questions such as: How fit is the immune system given a certain age? Would an immune boost be of therapeutic value, e.g. to improve the effectiveness of a simultaneous vaccination? Understanding the processes of immune system aging and degradation may also help in development of therapies that reverse some of the damages caused thus improving life expectancy. Therefore as a first step our research focuses on $\mathrm{T}$ cells; major contributors to immune system functionality. One of the main factors influencing immune system aging is the output rate of naive T cells. Of further interest is the number and phenotypical variety of these cells in an individual, which will be the case study focused on in this paper.
\end{abstract}

\section{INTRODUCTION}

System dynamics (SD) is an approach to help understand the behaviour of complex systems over time. It works with feedback loops, stocks and flows that help describe a system's nonlinearity. The methodology of developing SD models suggest some stages that should be accomplished in order to reach the final model. The first stage is identification of the problem followed by development of a dynamic hypothesis explaining its causes. The next stages are building the computational model, testing, validation and implementation.

Agent based modelling (ABS) is concerned with models in which agents interact with each other, with a view to observing their behaviors given changes to the environment.

This work compares SD and ABS immune system aging models that involve interactions which influence the naive $\mathrm{T}$ cell populations over time. The models are based on the mathematical equations defined in [9]. In their work, Murray et al. [9] propose a model with a set of equations to fit observed data and estimate the likely contribution of each of the naive $\mathrm{T}$ cell repertoire maintenance method.
In our work, we converted the original model into simulations and observe other important points not explored in [9]. Our objective is to understand the suitability of simulation for immune system aging problems. Moreover, we want to establish a comparison between the SD and ABS results, in terms of accuracy and complexity. In order to understand the problem and how the model was created, some concepts involving the aging of the immune system will be presented.

This paper is organized as follows. Section 2 presents the related work review, section 3 presents the immunological concepts necessary for the understanding of the modelled problem. Next, in Section 4, the conceptual and mathematical model are presented. Sections 5 and 6 present, respectively, the system dynamics and the agent based models, followed by the results obtained, conclusions and next steps of this ongoing work.

\section{RELATED WORK}

In this section, we briefly present some of the research literature related to the comparison of ABS with SD.

In [15], the authors show the application of both SD and ABS to simulate non-equilibrium ligand-receptor dynamics over a broad range of concentrations. They concluded that both approaches are powerful tools and are also complementary. In addition, in their case study, they did not indicate a preferred paradigm, although SD is an obvious choice when studying systems at a high level of aggregation and abstraction, and ABS is well suited to studying phenomena at the level of individual receptors and molecules.

In [11] the authors present a cross-study of SD and ABS. They define their features and characteristics and contrast the two methods. In addition they present ideas of how to integrate both approaches. The theoretical work presented by [10] compares ABS and SD conceptually, and discusses the potential synergy between them in order to solve problems of teaching decision-making processes.

The work presented in [13] also compares these modelling approaches and identify a list of likely opportunities for crossfertilization. The list presented is not exhaustive and should be a starting point to other researchers to take such synergistic views even further.

In our work, we intend to proceed with this investigation using as case studies simulations related to the aging of the 
immune system. In the next section we present concepts necessary to understand our case study.

\section{BACKGROUND}

Aging is a complex process that negatively impacts on the development of the immune system and its ability to function [1]. The changes that characterise the aging of the immune system are called collectively immunosenescence or decrease in immunocompetence.

The decrease of immunocompetence in the elderly can be envisaged as the result of the continuous challenge of the unavoidable exposure to a variety of viruses, bacteria, food and other harmful substances [6]. These exposures cause persistent life-long immune system stress, responsible for filling of the "immunological space" by an accumulation of immune cells and immune memory cells [6].

With age, there is also a significant reduction of certain specific kinds of immune cells, such as naive $\mathrm{T}$ cells, caused by the shrinkage of the organ that generates them, i.e. the thymus. Naive T cells are able to respond to novel pathogens that the immune system has not yet encountered. Lower numbers of these cells eventually leave the body more susceptible to infectious and non-infections diseases [9].

Before 20 years of age, naive T cells are sustained primarily from thymic output [9]. However, in middle age there is a change in the source of naive T cells; as the thymus involutes, there is considerable reduction in its $T$ cell output. Thus, new $\mathrm{T}$ cells are mostly produced by peripheral expansion. There is also a belief that some memory cells have their phenotype reverted back to naive cells [9]. Over time, the repertoire of naive $\mathrm{T}$ cells shrinks proportionately to faced threats, while memory increases [9] [8] [6].

Thus, late in life, the $\mathrm{T}$ cell population becomes less diverse and a small number of antigen-specific $\mathrm{T}$ cell clones can grow to a great percentage of the total $\mathrm{T}$ cell population. This takes over the space needed for other $\mathrm{T}$ cells, resulting in a less diverse and ineffective immune system. Eventually, there might be not enough naive $\mathrm{T}$ cells left to mount an effective defense and the immunological space is filled with memory cells.

The aim of this work is to develop models considering each of the above characteristics in order to investigate if these simulation techniques are suitable for investigating the immunosenescence phenomenon. De Martinis [8] and Franceschi [6] state that the most important characteristics of immunosenescence are the accumulation of memory $\mathrm{T}$ cells, reduction of naive $\mathrm{T}$ cells, shrinkage of the thymus and a filling of immunological space.

As we can see from the above, one of the main factors in the process of immunosenescence is the number and phenotypical variety of naive $\mathrm{T}$ cells in an individual, which changes with age in quantity and diversity. Recent research [2][5][7] suggests that population-based models of
T-cell repertoire evolution may guide new developments for treating diseases and help the recovery of the system after depletion caused by infections, radiation and age. These factors influenced the choice of the simulation case study developed in this research. Some work [14] has been done in simulation to understand certain aspects of immunology and other biological tissue patterns. However relatively little has been done in terms of immune degradation. Hence, our conceptual model considers shrinkage of the thymus and depletion of naive T cells, which will be explained in the next section.

\section{THE MODEL}

\subsection{Naive T Cell Output}

Thymic contribution in an individual are quantified by the level of a biological marker called ' $\mathrm{T}$ cell receptors excision circle' (TREC). TREC is circular DNA originated during the formation of the T-cell receptor. The percentage of $\mathrm{T}$ cells possessing TRECs decays with shrinkage of thymic output and activation and reproduction of naive $T$ cells [9]. This means that naive $\mathrm{T}$ cells originating from the thymus have a greater percentage of TREC than those originating through other proliferation.

Our model proposed here is based on data and equations obtained in [9], which is concerned with establishing an understanding naive $\mathrm{T}$ cell repertoire dynamics. The objective of the model is to determine the likely contribution of each of the naive T cell's sources by comparing estimates of the presence of TREC in the cells (see Figure 1). The dynamics of the sustaining sources, i.e. naive proliferation, TREC and reversal of memory to naive $\mathrm{T}$ cells are modelled mathematically.

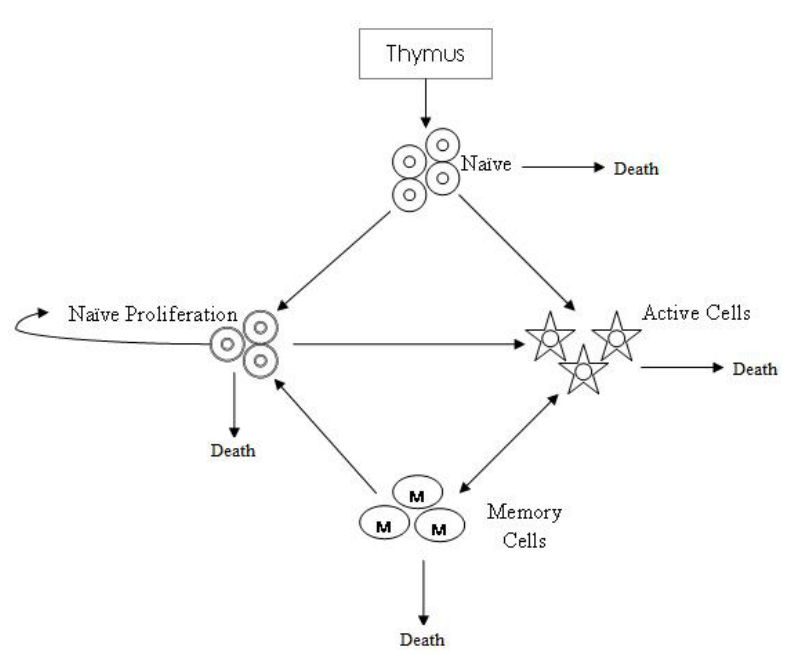

Figure 1. Dynamics of Naive T cells. 


\subsection{The Mathematical Model}

The mathematical model proposed in [9] is described by equations (1) to (6) below. In these equations, $N$ is the total number of naive cells of direct thymic origin, $N_{p}$ is the number of naive cells that have undergone proliferation, $A$ is the number of activated cells, $M$ is the number of memory cells and $t$ is time (in years). The first differential equation is:

$$
\frac{d N}{d t}=s_{0} e^{-\lambda_{t} t} s\left(N_{p}\right)-\left[\lambda_{n}+\mu_{n} g\left(N_{p}\right)\right] N
$$

where: $s_{0}$ is the thymic output; $\lambda_{t}$ is the thymic decay rate; $s_{0} e^{-\lambda_{t} t} s\left(N_{p}\right)$ represents the number of cells that arise from the thymus where $s\left(N_{p}\right)$ is the rate of export of the thymus defined by:

$$
s\left(N_{p}\right)=\frac{1}{1+\frac{\bar{s}^{N p}}{\bar{N}_{p}}}
$$

Also, $\lambda_{n} N$ represents the naive cells' incorporation into the naive proliferating pool where $\lambda_{n}$ is the naive proliferation rate; $\mu_{n}$ is the thymic naive cells death rate; $\mu_{n} g\left(N_{p}\right) N$ represents the naive cell death rate where the function $g\left(N_{p}\right)$ is the death rate of between naive TREC-positive and naive TRECnegative, defined as:

$$
g\left(N_{p}\right)=1+\frac{\frac{b N_{p}}{\overline{N_{p}}}}{1+\frac{N_{p}}{\overline{N_{p}}}},
$$

$\bar{N}_{p}$ and $\bar{s}$ are equilibrium and scaling values respectively. The second differential equation is:

$$
\frac{d N_{p}}{d t}=\lambda_{n} N+\left[\operatorname{ch}\left(N, N_{p}\right)-\mu_{n}\right] N_{p}+\lambda_{m n} M
$$

where: $c$ is the proliferation rate; $\operatorname{ch}\left(N, N_{p}\right) N_{p}$ represents the naive proliferation where $h\left(N, N_{p}\right)$ is the dilution of thymicnaive through proliferation defined by:

$$
h\left(N, N_{p}\right)=\frac{1}{1+\frac{N+N_{p}}{\bar{N}_{p}}}
$$

$\mu_{n} N_{p}$ is the death rate of proliferation-originated naive cells and $\lambda_{m n}$ is the reversion rate from memory into $N_{p}$. The final differential equation is:

$$
\frac{d M}{d t}=\lambda_{a} A-\mu_{m} M-\lambda_{m n} M .
$$

where: $\lambda_{a}$ is the reversion rate into memory and $\mu_{m}$ is the death rate of memory cells. The parameter values for the model's can be seen in Table 1 .

For the mathematical model and subsequent simulations, $s 0=56615$ and the active cells were obtained by a look-up

\begin{tabular}{|l|l|}
\hline rate & value $(\mathrm{s})$ \\
\hline \hline$\lambda_{t}$ & $\frac{\log (2)}{15.7}\left(\right.$ year $\left.^{-1}\right)$ \\
\hline$\lambda_{n}$ & $0.22,2.1,0.003$ \\
\hline$\mu_{n}$ & 4.4 \\
\hline$c$ & 0 (no proliferation) or $\mu_{n}\left(1+\frac{300}{\bar{N}_{p}}\right)$ \\
\hline$\lambda_{m n}$ & 0 \\
\hline$\mu_{m}$ & 0.05 \\
\hline$\lambda_{a}$ & 0 \\
\hline
\end{tabular}

Table 1. Rate values for the mathematical model.

table based on data collected by [3]. This table contains the number of activated CD4 cells per $\mathrm{mm}^{3}$ for early years. This table was used as a stock for the active cells. From the active cell stock we update the values of the memory cell stock.

Equations (1) to (6) will be incorporated in the SD and ABS models in order to investigate if it is possible to reproduce and validate the results obtained in [9]. Moreover, variations of the ratio variables are investigated to understand the importance of each individual integrand in the system. For example, it is important to establish how much the proliferation rate impacts the depletion of naive T cells over age; also, at which point in time can the system be defined as losing functionality.

\section{THE SYSTEM DYNAMICS MODEL}

The system dynamics model objective is to simulate the processes involved in the maintenance of naive $\mathrm{T}$ cells. The model was built taking into consideration all the interactions described by the mathematical equations defined in the previous section. The simulation was built using AnyLogic 6.5 University Edition [12].

The naive $\mathrm{T}$ cells, naive $\mathrm{T}$ cells from proliferation and memory cells are stock variables. The stock variable that represents the number of naive $T$ cells is subject to thymic output, proliferation and death rates, which are flow variables. The flows between naive cells and active cells are not defined in the mathematical model. Therefore we assume that these flows only interfere on the stock of active cells, which will not be considered in the SD model. The number of active cells, which is a stock, will be given by a look up table containing real values of active cells in the organism. The graphical representation of the stock of naive $\mathrm{T}$ cells and its flows, which corresponds to Equation 1 on the mathematical model, can be seen in Figure 2.

The amount of naive cells from proliferation is changed by death, proliferation and reversion from memory rates, according to Equation 2. The memory repertoire is modified by death and reversion to a naive phenotype (Equation 6). All the stocks put together form the SD model and can be seen in Figure 6. 


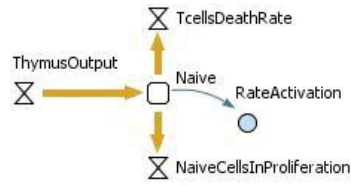

Figure 2. The naive $\mathrm{T}$ cell stock variable and its flows: thymic output, proliferation, activation and death.

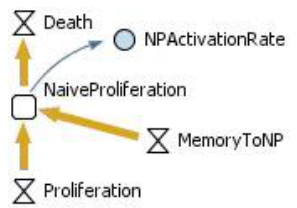

Figure 3. The naive $\mathrm{T}$ cells from proliferation stock variable and its flows: death, activation, proliferation and memory reverted to naive.

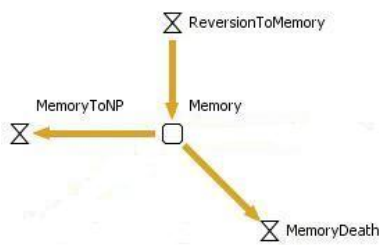

Figure 4. The memory $\mathrm{T}$ cells stock variable and its flows: death and reversion to a naive phenotype.

\section{THE AGENT BASED MODEL}

The SD model was then converted into an ABS model shown in Figure 7. $\mathrm{T}$ cells are the agents and the state chart represents all the stages these agents can assume, i.e. naive, naive from proliferation, active or memory cells. The agents' state changes and their death rates are given by the ratios defined in the mathematical model. Initially all the agents are in the naive state. As the simulation proceeds they can assume other stages according to the transition pathways defined in the state chart. The agents also reproduce, and the newborn agents which are also T cells, should assume the same state as their original agent.

At the beginning of the simulation, there are only agents in the Naive state generated by thymic output. From the Naive state, the agents can proliferate and go to the NaiveProliferation state. If the T cells in the NaiveProliferation state reproduce according to the NaiveProliferationRate, more agents will be generated in the same state (NaiveProliferation) as their progenitors. The same thing happens to the state Memory. The agents can also die according to specific rates, determined by the mathematical model. The agents in this simulation respond to changes in time and do not interact with each other directly. Therefore, what we intended to get from

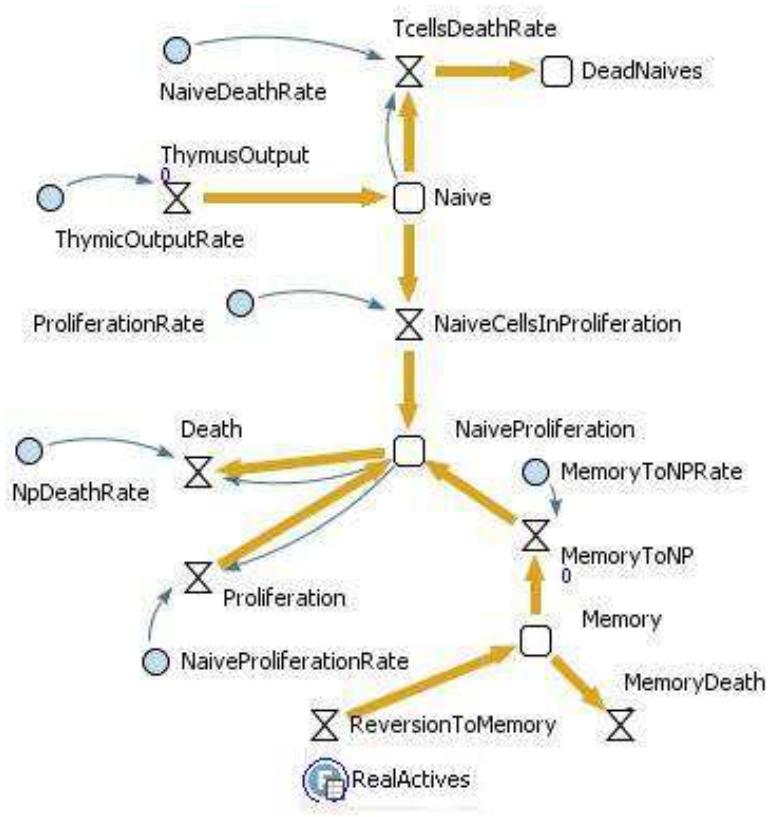

Figure 5. The system dynamics model's functions and parameters. The function RealActives returns the values for active CD4+ cells at a certain time. The parameters NPActivationRate, NPActivation, NPDeathRate, MemoryToNP and MemoryToNPRate correspond to naive cells from proliferation (NP).

this simulation is the response of each individual cell to the proposed environment.

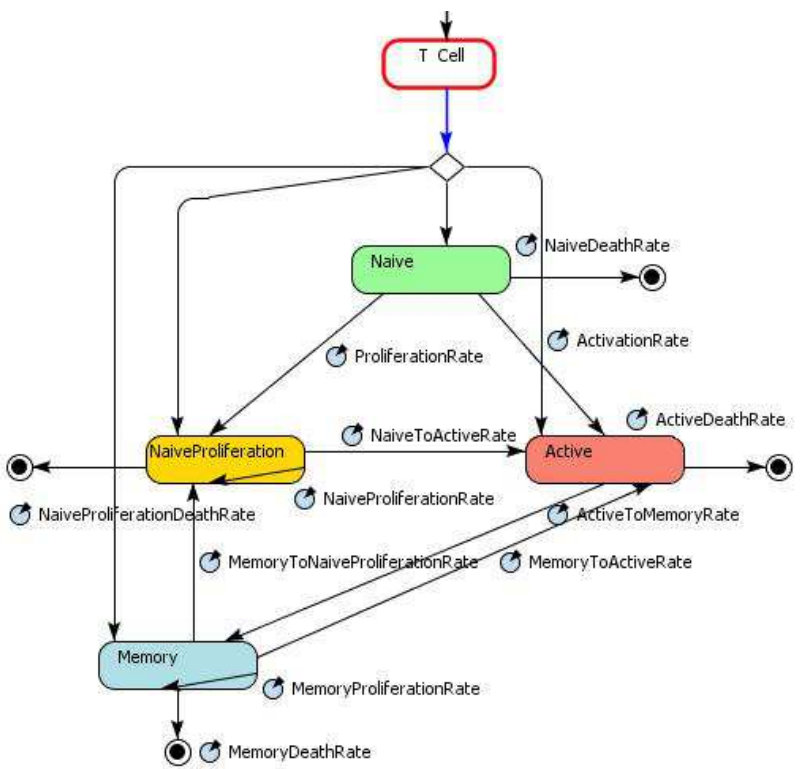

Figure 6. The ABS model. In this simulation, The number of active agents is also given by a look up table. 


\section{EXPERIMENTS}

We study three simulation scenarios defined by [9] with different values for the parameters. A summary of parameters changed for each scenario can be seen in Table 2 .

One of the case study simulation's objectives is to investigate if there is the need for naive peripheral proliferation throughout life. Therefore, for the first scenario the naive peripheral proliferation rate is set to zero. It also considers reversion from memory to a naive phenotype.

The second scenario assumes peripheral proliferation with a bigger rate of naive cells becoming naive proliferating cells (2.1): There is no reversion from memory to a naive phenotype and no homeostatic reduction in thymic export. The functions $s, g$ and $h$ from the mathematical model are responsible for controlling the thymic export, naive death rate and naive peripheral proliferation, respectively. In order to alter the thymic export, the parameters $\bar{s}$ and $\bar{N}_{p}$ have been changed. The parameter $b$ was set to zero so that the function $g$ would remain constant during all the simulation and so the death rate of naive cells.

The third scenario alters the function $g$ over time by setting the parameter $b$ greater than zero $(b=4.2)$. This means that the death rate of naive $\mathrm{T}$ cells from thymus will increase along the years as the number of naive from peripheral proliferation increases. There is no change of the thymic export, no reversion from memory to a naive phenotype and the conversion rate of naive from thymus to naive proliferation is low (equal to 0.003).

The data used for validation of the mathematical model and the simulations is displayed in Table 3. The data set contains information about the TREC marker in individuals grouped in age ranges. The first column of Table 3 shows the age range of the individuals; the second column has the mean $\frac{\log _{10} \text { TREC }}{10^{6}}$ PBMC (peripheral blood mononuclear cell) and the third column contains the number of individuals in each age range. The total number of individuals in the experiment was 506. The graphic containing the TREC data (naive from thymus) and total naive cell data provided by [4] is shown in Figure 8.

Each simulation was run for a period of one hundred years considering the impact of thymic shrinkage per $\mathrm{mm}^{3}$ of peripheral blood and 3673 initial naive cells from thymus for the SD model.

\section{RESULTS}

The SD simulation results for the first scenario can be seen in Figure 9. The ABS results are shown in Figure 10. The results for both simulation techniques show a very similar trend curve, although the ABS results exhibit a more noisy behavior in time. For this first scenario, the simulation results did not

\begin{tabular}{|l|l|l|}
\hline Age & $\frac{\log _{10} \text { TREC }}{10^{6} \times n}$ PBMC & number of individuals \\
\hline \hline 0 & 5.03 & 48 \\
\hline $1-4$ & 4.93 & 53 \\
\hline $5-9$ & 4.86 & 19 \\
\hline $10-14$ & 4.86 & 19 \\
\hline $15-19$ & 4.56 & 33 \\
\hline $20-24$ & 3.88 & 26 \\
\hline $25-29$ & 3.75 & 47 \\
\hline $30-34$ & 3.61 & 65 \\
\hline $35-39$ & 3.54 & 73 \\
\hline $40-44$ & 3.52 & 52 \\
\hline $45-49$ & 3.37 & 55 \\
\hline $50-54$ & 3.17 & 16 \\
\hline
\end{tabular}

Table 3. The dataset used for validation obtained in [9].

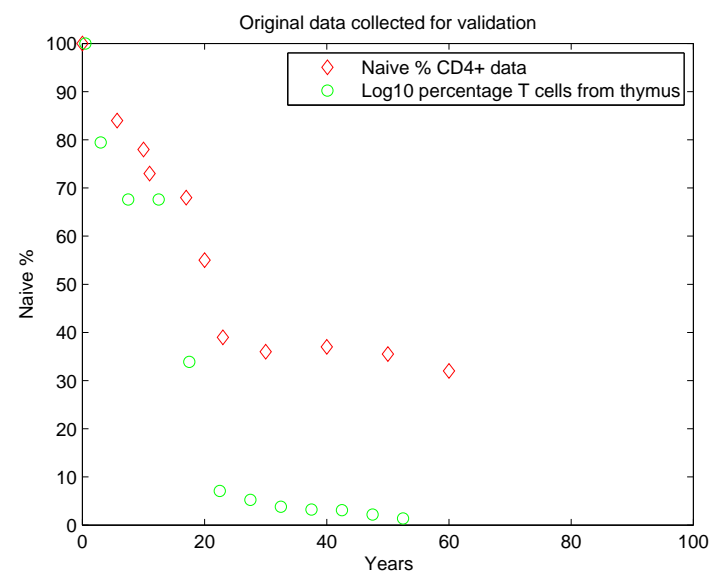

Figure 7. The dataset used for validation was provided by [9] and [4]. The data represented by the symbol $\circ$ is the TREC data shown in Table 3 and $\diamond$ represents the total percentage of CD4+.

fit the original data. The naive cells from thymus curve produced shows a big decay in thymic export on the beginning of life because of the high death rate.

After the twenties, an exponential decay of thymic export is observed and the dynamics follows the thymic decay rate rule defined in the mathematical model. The naive proliferation curve increases with the decrease of naive from thymus, but as there is no proliferation of peripheral cells, they die with no replacement. Thus they follow the same pattern of their only source, i.e. thymic naive cells. The results indicate that peripheral proliferation is important for maintenance of naive T cells.

For scenario 2, results match the original data more closely than in the previous scenario. In this case peripheral proliferation is considered as well as a high rate of naive cells from the thymus turning into peripheral naive cells. Figure 11 shows 


\begin{tabular}{|l|l|l|}
\hline Scenario & Description & Parameters \\
\hline \hline 1 & No peripheral proliferation & $c=0, \lambda_{n}=0.22, \lambda_{m n}=0.05, \bar{N}_{p}=387, \bar{s}=0.48, b=3.4, \mu_{N p}=0.13$ \\
\hline 2 & $\begin{array}{l}\text { No homeostatic reduction in thymic export, } \\
\text { no homeostatic alteration of naive death rate }\end{array}$ & $\bar{s}=0, b=0, \lambda_{n}=2.1, \lambda_{m n}=0, \bar{N}_{p}=713$ \\
\hline 3 & $\begin{array}{l}\text { Homeostatic alteration of naive death rate } \\
\text { but not thymic export }\end{array}$ & $\bar{s}=0, \lambda_{n}=0.003, \lambda_{m n}=0, \bar{N}_{p}=392, b=4.2$ \\
\hline
\end{tabular}

Table 2. Simulation parameters for different scenarios.

the results for SD and Figure 12 for ABS. The naive from thymus curve shows a big decay in the beginning of life because of the death and proliferation rates. On the other hand, the naive from proliferation curve increases with the decrease of the naive from thymus curve. This pattern is controlled by the $g$ function.

The main difference between these results and results form the previous scenario is that the amount of naive cells from proliferation reaches a stable value after the age of twenty with no further decay. The results indicate the importance of peripheral expansion, but the need of a smaller rate of naive to peripheral naive conversion. Moreover, reversion from memory is not important.

Scenario 3 takes into account the results produced in the previous scenarios and adjusts the parameters in a way that a more accurate output is obtained. The results for SD and ABS can be seen in Figures 13 and 14, respectively. The naive from thymus curve presents a decay at the beginning of life followed by an interval of stability ruled by $s 0$. By the age of twenty the thymic export decreases in an exponential trend. With the decay of naive from thymus, the naive repertoire changes from the thymic source to peripheral proliferation source. Therefore, by performing these simulations it is possible to have an idea of how the decay of naive cells happens over time. The results now closely match the original data.

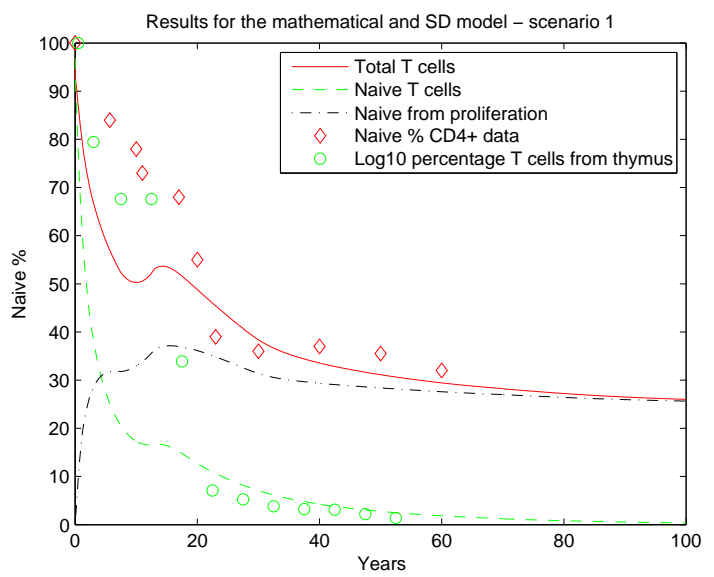

Figure 8. Results for scenario 1 with SD.

For the three scenarios studied, the simulations produced

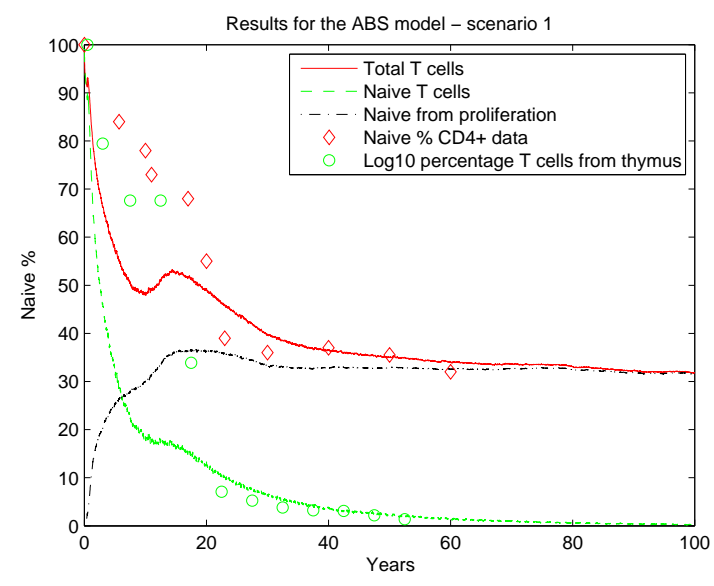

Figure 9. Results for scenario 1 with ABS.

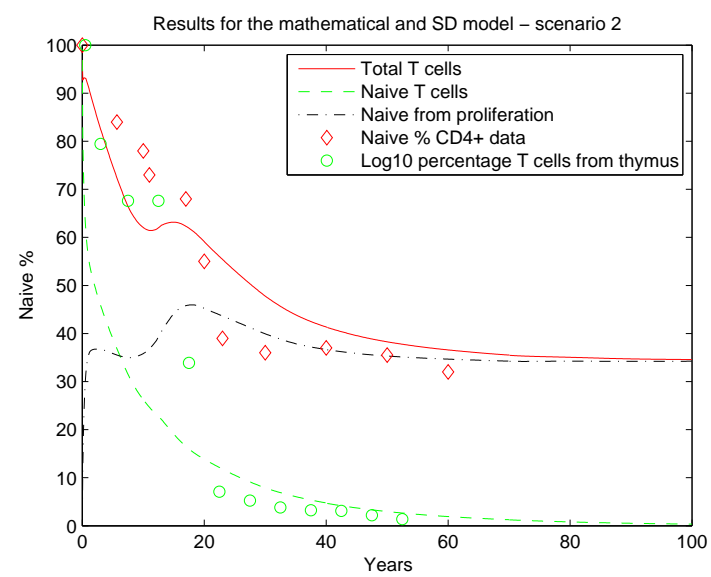

Figure 10. Results for scenario 2 with SD.

similar results for both $\mathrm{SD}$ and $\mathrm{ABS}$ techniques matching the original data. Hence, it is possible to conclude that the mathematical model, as well as immunosenescence problems are suitable for simulation using both techniques. The differences between the SD and ABS simulations are in how the conceptual model is represented and manipulated.

SD gives a systemic view of the conceptual model and tries to forecast how the system as a whole would evolve in time in an aggregate manner. This means that each change ratio will 


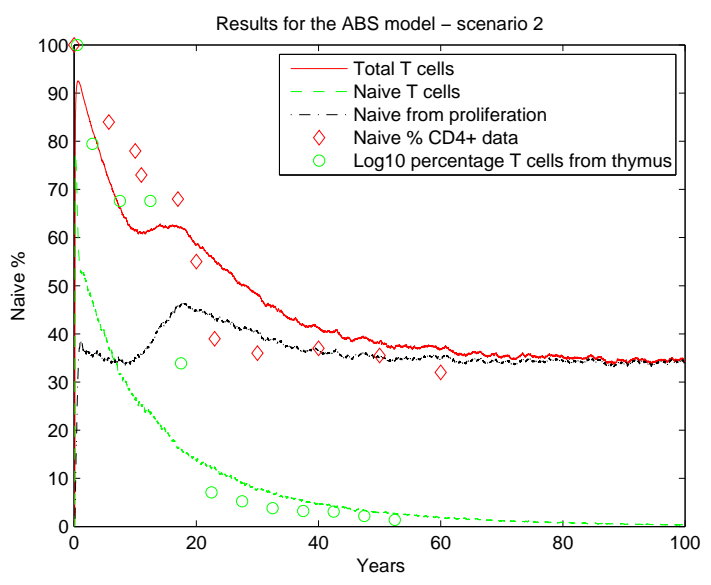

Figure 11. Results for scenario 2 with ABS.

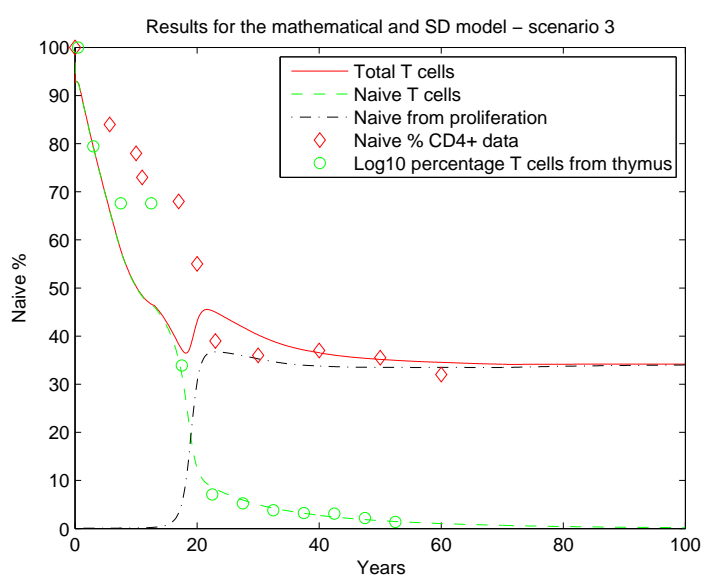

Figure 12. Results for scenario 3 with SD.

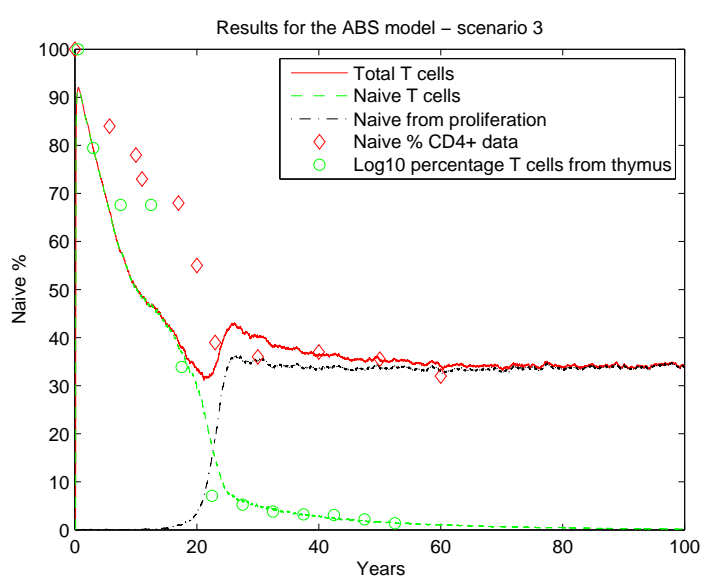

Figure 13. Results for scenario 3 with ABS. be applied to the entire set of cells. One could argue that SD represent an idealised version of the system.

In the ABS model, cells are subject to individual rates that occur during the time slice they were created in. This makes the output more noisy than the SD results. It also seems to be more realistic, because in real immune systems, cells have individual behaviours and responses to the environment. In addition, the ABS model is easier to adapt to changes in the conceptual model, such as when change rates occur during a cell's life span or new cellular interactions between cells.

We also believe that the individual cellular behavior could be captured in a more simplified mathematical model. For example, the $s, g$ and $h$ functions' control could be incorporated in the way cells change over time. This would help to further understand the biological system behavior. On the other hand, $\mathrm{SD}$ is simpler to implement and demands less computational resources such as memory, processing time and complexity as it can be seen in the comparison of Tables 4 and 5. The precision measure on the table is the sum of the square errors (SSE) of the percentages of naive $\mathrm{T}$ cells from thymus. The best results are given by the smaller numbers of SSE. The other measurements are time in seconds and memory usage in megabytes. The numbers for the system dynamics are better in terms of resources consumption as well as precision. We believe that precision for the ABS was not better because of the instabilities that occurred in the resulting graphs.

\begin{tabular}{|l|c|c|c|}
\hline Scenario & Time $(\mathrm{sec})$ & RAM & SSE \\
\hline 1 & 0.7 & 11 & 71696 \\
\hline 2 & 0.8 & 11 & 5924.4 \\
\hline 3 & 0.7 & 10 & 589.9 \\
\hline
\end{tabular}

Table 4. SD processing time, memory usage and precision.

\begin{tabular}{|l|c|c|c|}
\hline Scenario & Time $(\mathrm{sec})$ & RAM $(\mathrm{MB})$ & SSE \\
\hline 1 & 28800 & 40 & 10349 \\
\hline 2 & 61700 & 50 & 20668 \\
\hline 3 & 64800 & 50 & 45834 \\
\hline
\end{tabular}

Table 5. ABS processing time, memory usage and precision.

\section{CONCLUSIONS}

Understanding immunosenescence and its causes may help direct research into therapies that reverse some of its consequences and improve life expectancy. The research question raised within this work focuses on the feasibility of using SD and ABS simulation tools to understand immune system aging and comparing the two strategies. The ability to model immune system aging will provide new means to define a patient-specific 'Immune Risk Phenotype'. Modelling this Risk Type will improve our knowledge of immune factors that contribute to morbidity and mortality and facilitate the design of appropriate interventions. 
The main factors influencing the process of immunosenescence include the number and phenotypical variety of naive $\mathrm{T}$ cells in an individual, which change with age in quantity and diversity. At the beginning of life, the thymus is the principal source of naive T cells. With age, there is a decay in thymus output and a shift between the main source of naive $T$ cells. It is believed that the sustenance of naive $\mathrm{T}$ cells in the organism is provided by peripheral expansion, reversion from a memory phenotype, and long-lived T cells. Data related to thymic output rate was collected by [9] and a mathematical model was build. This mathematical model was used as the baseline for the development of SD and ABS models.

Three simulation scenarios were studied and the simulation outputs were broadly similar for both SD and ABS. Hence, it is possible to conclude that the mathematical model, as well as immunosenescence problems are suitable for simulation using both techniques. The differences between the SD and ABS outputs are in how the conceptual model is represented and manipulated.

The SD based simulation is closer to the underlying mathematics, but has the disadvantage of being high-level, with complete homogeneity of simulated entities. On the other hand, ABS allowed a representation of each entity and heterogeneity. However, ABS increases the demand for computational resources.

As future work, it is intended to build a framework to develop immunosenescence simulation models. We also want to define guidelines to help decide when each simulation type should be used in an immunosenescence model.

Finally, we are interested to investigate how combining ABS and SD models could help improving the simulations. As we have shown, immunosenescence can be simulated by more than one simulation type, however our hypothesis is that a combination of methodologies will provide higher prediction accuracy given the same amount of input data and computational resources.

\section{REFERENCES}

[1] Matteo Bulatti, Mariavaleria Pellicanò, Sonya Vasto, and Guiseppina Colonna-Romano. Understanding ageing: Biomedical and bioengineering approaches, the immunologic view. Immunity \& Ageing, 5(9), september 2008.

[2] Michael P. Cancro, Yi Hao, Jean L. Scholz, Richard L. Riley, Daniela Frasca, Deborah K. Dunn-Walters, and Bonnie B. Blomberg. B cells and aging: molecules and mechanisms. Trends in Immunology, 30(7):313-318, July 2009.

[3] W. Marieke Comans-Bitter, Ronald de Groot, and René van den Beemd. Immunophenotyping of blood lymphocytes in childhood. reference values for lymphocites subpopulations. $J$. Pediatr., (130):388-393, 1997.

[4] Andrea Cossarizza, Claudio Ortolani, Roberto Paganelli, Daniela Barbieri, Daniela Monti, Paolo Sansoni, Umberto Fagiolo, Gastone Castellani, Ferdinando Bersani, Marco Londei, and Claudio Franceschi. CD45 isoforms expression on CD4+ and CD8+ T cells throughout life, from newborns to centenarians: implications for T cell memory. Mechanisms of Ageing and Development, 86(3):173 - 195, 1996.

[5] Mark R. Dowling and Philip D. Hodgkin. Why does the thymus involute? a selection-based hypothesis. Trends in Immunology, 30(7):295-300, July 2009.

[6] Claudio Franceschi, Massimiliano Bonafè, and Silvana Valensin. Human immonosenescence: the prevailing of innate immunity, the failing of clonotypic immunity, and the filling of immunological space. Vaccine, 18:1717-1720, 2000.

[7] Elizabeth J. Kovacs, Jessica L. Palmer, Carl F. Fortin, Tamas Fulop Jr, Daniel R. Goldstein, and Phyllis-Jean Linton. Aging and innate immunity in the mouse: impact of intrinsic and extrinsic factors. Trends in Immunology, 30(7):319-324, July 2009.

[8] Massimo De Martinis, Claudio Franceschi, Daniela Monti, and Lia Ginaldi. Inflamm-ageing and lifelong antigenic load as major determinants of ageing rate and longevity. FEBS, 579:2035-2039, february 2005.

[9] John M. Murray, Gilbert R. Kaufmann, Philip D. Hodgkin, Sharon R. Lewin, Anthony D. Kelleher, Miles P. Davenport, and John Zaunders. Naive T cells are maintained by thymic output in early ages but by proliferation without phenotypic change after twenty. Immunology and Cell Biology, 81:487495, June 2003.

[10] John Pourdehnad, Kambiz Maani, and Habib Sedehi. System dynamics and intelligent agent based simulation: where is the synergy? In Proceedings of the XX International Conference of the System Dynamics society., 2002.

[11] Nadine Schieritz and Peter M. Milling. Modeling the forrest or modeling the trees: A comparison of system dynamics and agent based simulation. In Proceedings of the XXI International Conference of the System Dynamics society., 2003.

[12] XJ Technologies Simulation Software and Services. Anylogic Muti-Method Simulation Tool, Available: http://www.xjtek.com/anylogic/download/, Last accessed 02 Jun 2010

[13] Luminita Stemate, Ivan Taylor, and Codrin Pasca. A comparison between system dynamics and agent based modeling and opportunities for cross-fertilization. In Proceedings of the 2007 Winter Simulation Conference. S. G. Henderson, B. Biller, M.-H. Hsieh, J. Shortle, J. D. Tew, and R. R. Barton, 2007.

[14] Bryan C. Thorne, Alexander M. Bailey, and Shayn M. Peirce. Combining experiments with multi-cell agent-based modelling to study biological tissue patterning. Briefings in Bioinformatics, 8(4):245-257, may 2007

[15] Wayne W. Wakeland, Edward J. Gallaher, Louis M. Macovsky, and C. Athena Aktipis. A comparison of system dynamics and agent-based simulation applied to the study of cellular receptor dynamics. Hawaii International Conference on System Sciences, 3:30086b, 2004 